\title{
Nurses as information providers: facilitating understanding and communication of statistical information
}

\author{
Yaniv Hanoch ${ }^{* 1}$, Thorsten Pachur
}

Max Planck Institute for Human Development, Center for Adaptive Behavior and Cognition, Lentzeallee 94, Berlin 14195, Germany

Accepted 15 January 2004

\author{
KEYWORDS \\ Decision making; \\ Natural frequencies; \\ Genetic counselling; \\ Information providers; \\ (in) Numeracy; \\ Probabilities; \\ Screening
}

\begin{abstract}
Summary Nurses are increasingly being called upon to be the conveyers of important statistical information to patients. This trend is particularly evident in the domains of genetics and cancer screening. These new roles, however, demand new competencies, such as the ability to solve statistical problems, and the skill to communicate the answers effectively, as effective communication is an important ingredient in shared decision making. Genetic testing, perhaps more than other medical domains, relies heavily on the use of statistics. Being able to convey statistical information effectively is vital. In this paper, we illustrate the problems health care professionals have had in tackling and communicating statistical information. We introduce the natural frequencies method of solving Bayesian inference problems and review empirical evidence that shows the superiority of this format. Being able to transform probabilities into natural frequencies facilitates correct Bayesian inferences. It is argued that the conventional approach to educating nurses in Bayesian problem solving should be reconsidered and their statistical curriculum should be supplemented with instruction in using the natural frequency format.

() 2004 Elsevier Ltd. All rights reserved.
\end{abstract}

\section{Introduction}

Patients are increasingly seeking, and are in fact expected, to be involved in the decision process concerning medical procedures, screenings, and

\footnotetext{
"Corresponding authors. Tel.: +49-30-824-06-249; fax: +49-30824-06-394/+49-30-8249939.

E-mail address: pachur@mpib-berlin.mpg.de (T. Pachur).

1 Present address: Department of Health Services, UCLA School of Public Health, $650 \mathrm{~S}$. Young Drive, Los Angeles, CA 90095-1772, USA. Tel.: +1-310-825-2594; fax: +1-310-825-3317.
}

treatments they are offered (Lidz et al., 1984; Wear, 1993). To make competent decisions patients need information, including knowledge of the risks and benefits associated with each medical option. How this knowledge is imparted typically leaves the patient with some degree of uncertainty: What do the success rates of a given treatment mean for me? What are the risks involved with a particular screening intervention, and how likely am I to suffer these consequences? For patients to be able to evaluate the nature of each treatment option, it is crucial that health 
care providers effectively process quantitative information and efficiently and effectively communicate it to patients (Schwartz et al., 1997). In addition, to ensure evidence-based practice, health care professionals must be competent in evaluating research studies, which traditionally hinge on the concept of probability (Taylor and Muncer, 2000).

Statistical information, however, has been plaguing the health care profession: numerous investigators have shown that health care providers encounter difficulties in understanding and transmitting such information. Most researchers have tended to focus on the patients' (Newell and Vogel, 1988 ) or on the physicians' aptitudes (Gigerenzer and Hoffrage, 1995) while largely neglecting to examine nurses' competence (see McManus et al., 2002).

In this paper, we capitalize on previous psychological and medical research to highlight the necessity of nurses being skilled in understanding and solving statistical problems, on the one hand, and being able to disseminate the processed information effectively on the other. We offer one technique that can assist nurses in engaging in both tasks: understanding statistical information and transmitting it to patients.

\section{Nurses as information providers}

The delivery of health care in primary care settings is increasingly being undertaken by nurses and other health and social care workers. Nurses, especially, have experienced a major expansion in the scope of roles they play (Taylor, 2002; Tzeng and Ketefian, 2003; Valanis, 2000). They account for the single largest component of hospital staff, and current estimations project that employment for nurses will be the fastest growing occupation in the coming years (American Association of Colleges of Nursing n.d.). Nurses are becoming the primary providers of information previously seen as falling within the sole domain of doctors, in particular providing information relating to risk of disease, diagnosis, prognosis, and treatment options.

Although doctors have, and will no doubt continue to have, a significant role in providing information, it has been shown that a number of barriers - for example, short interaction time (e.g., Howie et al., 1999) and patient anxiety (Bush and Osterweis, 1978) - reduce the effectiveness of the interaction and communication between physicians and patients. As a result, the patients' ability to process, understand, and recall vital information can be impeded, contributing to pa- tients' low levels of recall of this information (Ley, 1982; Ellis and Tattersall, 1999). Nurses, on the other hand, arguably, form a more egalitarian relationship with patients and entertain less emotionally taxing interactions with them.

\section{Nurses as information providers: genetic testing and mammography screening}

The scope of nursing is rapidly expanding and specializing, often to territories where statistical information plays a key function. Genetic counseling and mammography screening are examples of such domains. While the upsurge in geneticrelated information serves as one motivation for this paper, we derive our findings from research on mammography screening (for a similar concern among radiographers, see Wivell et al., 2003).

With rapid developments in DNA technology over 800 genetic tests are currently available or being developed (see GeneTests-GeneClinics: http://www.geneclinics.org) - and with many known gene disorders, genetics is assuming a more dominant role in our understanding of disease diagnostics, treatment, and prevention. Given that the demand for genetic screening is constantly rising, coupled with a projected shortage of genetic counsellors, nurses are being called upon to assume the counseling role (Emery and Hayflick, 2001; DoH, 2003; Kirk et al., 2003). Accordingly, nurses will need to acquire up-to-date knowledge about human genetics, as they will function as one of the main sources of information for the patients, their families, and the communities that they serve (Lashley, 1999; Lea, 2000). Our principal concern stems from the fact that to offer genetic tests to their clients, genetic counselors need "adequate knowledge of genetics and genetic tests, including the ability to interpret probabilistic information" (Hofman et al., 1993, p. 625; emphasis added). The need for statistical knowledge and skills, therefore, will intensify as well. It has been shown, however, that health care professionals are struggling with the difficulties of interpreting probabilities in the context of genetic testing (Reyna, 2001).

Nurses will help patients make decisions about participating in clinical studies and signing informed consent forms (Williams, 1998); Pediatric nurses will advise parents about prenatal genetic screening (Lessick and Anderson, 2000); perioperative nurses will counsel patients in regard to genetic information (Lea and Tinley, 1998); and oncology nurses are already in the position "to 
translate [genetic] information to patients and their families and, ultimately, enhance comprehensive care through patient education and advocacy" (Stoltzfus et al., 2001, p. 201).

As in the case of mammography screening, which we discuss below, genetic testing is inherently associated with uncertainty: most tests do not always detect a disease and positive test results are also possible when the disease is absent. Thus, positive genetic test results predict the actual occurrence of a disease only with a particular probability. Uncertainty is thus present both in relation to patients' decisions to undergo testing, and, even more significantly, in interpreting and understanding the results and their ramification for the patients' (and their relatives') present and future behaviors (Evans et al., 2001). As quality genetic counseling and testing is heavily dependent on adequate risk communication, nurses face the challenge of expressing these complex sets of information - including the interplay between genetic makeup and environmental and lifestyle factors - meaningfully to patients (Croyle and Lerman, 1999). How do nurses handle this task, if at all, in their daily practice? What, if any, problems do they face, and what remedies can be offered?

\section{The problem of (in) numeracy}

As many health-related options are clouded in uncertainty, probabilistic information constitutes a crucial ingredient in making sound and informed medical decisions (Weinstein, 1999; Hallowell, 2000). There is little disagreement that nurses and other health care providers use probabilistic information (Hallowell et al., 1997). It is less clear, however, whether nurses, like other health professionals, are able to utilize this quantitative information effectively. As Maguire (1999) has argued, "few doctors and nurses receive sufficient training to ensure they are proficient in key communication tasks" (p. 2058).

In one study, Edwards et al. (1998) investigated, among other things, nurses' approaches to communicating risk information to patients and the barriers they encounter in the process. It was reported that numeric data such as probabilities were commonly used in risk communication, yet a major finding of this study was that the nurses (as well as the other health care professionals) encountered problems resulting from divergent interpretations of the probabilistic information by nurses and patients.
Although such problems might cause one to question the value of using numbers [There is an ongoing debate on whether statistical information should be expressed verbally (e.g., often, sometimes, etc.) or numerically. Our aim is not to provide an exhaustive review on the subject but to deal only with the numerical expression of uncertainty (Erev and Cohen, 1990; Hallowell et al., 1997)]; it is more instructive to consider what factors contribute to these difficulties. Numeracy is generally defined as the facility with which probabilities and basic mathematical concepts are understood (Schwartz et al., 1997). Lipkus et al. (2001) developed a numeracy scale to assess this competence and they found that, even among a "highly educated" sample, a large proportion of the subjects could not solve basic probability problems and had difficulties distinguishing magnitudes of 2, although these calculations are basic and elementary tasks in many decision aids (Lipkus et al., 2001). For instance, almost $80 \%$ of the subjects could not correctly perform the simple action of converting a chance of 1 in 1000 into percentages. Finding similar results, Schwartz et al. (1997) argued that an association exists between numeracy and the ability to evaluate the benefits inherent in health care choices, for example, undergoing genetic screening (although, in this study even numerate participants overestimated the benefits of screening). This led the authors to conclude that "current formats for presenting such information may be ineffective," and they suggested the "[development of] communication strategies that overcome innumeracy" (p. 972). In the next section we propose one such strategy. By altering the representation format typically used - namely from probabilistic to natural frequency - nurses will more easily be able to overcome some of the difficulties discussed.

\section{Improving statistical communication: the natural frequency way}

Nurses are already, and will continue to be, responsible for providing important information to patients - especially in areas where the medical establishment has not been able to voice a unified statement. We use mammography screening to illustrate our point, but similar considerations might be applicable to genetic testing. Olsen and Gøtzsche's (2001) recent analysis, arguing that mammography screening has failed to reduce mortality rate, has sparked a heated discussion over the usefulness and harms of early mammography 
screening. Thousands of women, after Olsen and Gøtzsche's findings were publicized in the New York Times and other popular press, felt unsure and bewildered by the conflicting messages, causing many of them to call their health care provider or cancer-related organization to inquire whether they should or should not be screened. The question women face is whether the benefits of screening outweigh the potential harms - after all, mammography and genetic testing are not without physical and/or psychological risks. These risks include false diagnosis of a disease, potential insurance and employment discrimination, stigmatization, interference and pressure from other family members, and diminished self-worth. It would not be a surprise, therefore, if women contemplating mammography screening (or genetic screening for breast cancer (BRCA) 1 and breast cancer (BRCA) 2 mutation for breast cancer) will pose the following question to their oncology or genetic counselor nurse: What is the probability that I have breast cancer, given that I have a positive mammography result?

What seems like a straightforward question at first glance might turn out to be a difficult one when it is actually confronted. Three kinds of data are needed to grasp the meaning of a test result: the base rate (prevalence) of the disorder; the probability that the test is positive if the disorder is present (sensitivity); and the probability that the test will yield a positive result in the absence of the disorder (false positive rate). This information, for example, was rated by both doctors and patients as the most essential in deciding whether to undergo prostate-specific-antigen (PSA) screening (Chan and Sulmasy, 1998). Yet health care professionals and laymen alike have serious difficulties integrating this information when asked for the positive predictive value (i.e., the probability that a person found to have a positive test result actually has the disease) of a test.

An early study by Casscells et al. (1978) with 60 house officers, medical students, and physicians from Harvard Medical School addressed this question. Subjects were asked to estimate the positive predictive value of a test, given only the prevalence and false positive rate. Subjects' estimates ranged so broadly (from $2 \%$ to $95 \%$ ), with many of them far off the mark, that the authors deduced that "formal decision analysis was almost entirely unknown and even common-sense reasoning about the interpretation of laboratory data was uncommon" (p. 1000). Based on these earlier findings, Eddy's (1982) informal study presented doctors with the following statistical information about mammography (we use the values $80 \%$ and $10 \%$ for the sensitivity and false positive rate for the sake of simplicity; in the original study the values $79.2 \%$ and $9.6 \%$ were used, respectively): (i) the probability that a patient has breast cancer is $1 \%$, (ii) the probability of obtaining a positive mammogram if the patient has cancer is $80 \%$, and (iii) the probability of obtaining a positive mammogram if the patients does not have cancer is $10 \%$. The doctors' task was to determine the probability that a patient with a positive mammogram actually has breast cancer (the positive predictive value). This task can be solved by inserting the figures into Bayes' rule. Eddy's (1982) results were extremely troubling, as 95 of 100 doctors arrived at the wrong answer, assuming it to be around $75 \%$, whereas the correct answer is one order of magnitude smaller, namely, $7.5 \%$. In that a variety of health professionals have failed to perform these calculations accurately, it seems reasonable to assume that nurses will face similar difficulties. Given these findings, one might conclude that information presented in a probability format - that is, prevalence, sensitivity, and false alarm rate as singleevent probabilities - is generally miscalculated by health professionals and as a consequence may be miscommunicated to patients (needless to say, others factors also hamper the communication process between health care professionals and patients).

Can we provide a solution to this daunting problem? Research has shown that presentation format matters: the presentation format used by health care professionals does influence patients' psychological states (e.g., hope and fear), their readiness to undergo screening procedures, and their appreciation of the risks and benefits associated with various medical procedures (Hoffrage et al., in press). The crucial point here is that the probability format is only one possible method of presenting statistical information.

In one study, Gigerenzer and Hoffrage (1995) provided subjects precisely the same kind of information as in Eddy's (1982) original study and asked them to calculate the probability of having a disease given a positive test result. Crucially, whereas in one condition the subjects received the information as single-event probabilities, in the other condition the subjects received the information as natural frequencies. More specifically, subjects in the latter condition were told the following (see also Fig. 1):

Ten out of every 1000 women at age 40 [...] have breast cancer. Eight out of 10 women with breast cancer will have a positive mammography. Ninety-five out of 990 women without breast cancer will also have a positive mammogram. 
The task subjects faced was to indicate how many women who have a positive mammogram in a routine screening actually have breast cancer. From a mathematical point of view, the natural frequency format and the probability format are equivalent. Yet, are they equivalent psychologically? Put differently, can we expect similar results irrespective of presentation format? The findings reported by Gigerenzer and Hoffrage (1995) show that presenting the diagnostic information as natural frequencies can tremendously increase people's ability to draw correct inferences: whereas only $16 \%$ of the subjects who were given the information as probabilities arrived at the correct solution, the proportion of correct solutions jumped to $46 \%$ when the same information was given in the natural frequency format.

Provided that the facilitating effect of natural frequencies hinges on their property of containing information about the base rates, it is important to point out the difference between natural frequencies and normalized frequencies (cf. Hoffrage et al., 2002). Normalized frequencies do not contain information about base rates and thus do not facilitate Bayesian reasoning, whereas natural frequencies result from counting the occurrences of events (i.e., having breast cancer, having no breast cancer, having a positive mammogram, having a negative mammogram) in a population of, say, 1000 (as in the tree in Fig. 1). In the normalized frequency format the events' frequencies are expressed in relation to an a priori fixed reference number (and not according to the frequencies ob-

\section{Natural Sampling}
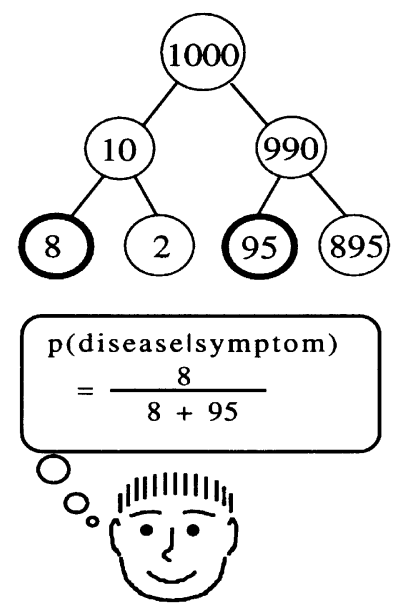

Standard Probability
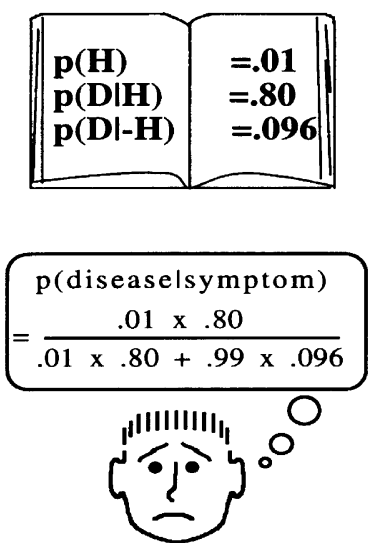

Figure 1 Bayesian inference and information representation (natural sampling of frequencies and standard probability format). Figure from Gigerenzer and Hoffrage (1995), reprinted with permission of the American Psychological Association. served in the reference population). Compare the representation in natural frequencies in Fig. 1 with the normalized frequency: "Ten in 1000 women have breast cancer. Eight hundred in 1000 women who have breast cancer will have a positive mammogram. One hundred in 1000 women who do not have breast cancer will also have a positive mammogram." This normalization eliminates information about the base rate.

Two arguments are generally made to explain the facilitating effect of natural frequencies (e.g., Gigerenzer and Hoffrage, 1995; Hoffrage et al., in press). First, the computational operations needed to solve a Bayesian inference problem are much easier when based on natural frequencies - as the number of computational steps is smaller (see Fig. 1) - than when they are based on probabilities. This is because, in contrast to single-event probabilities, natural frequencies already carry information about base rates in a population (i.e., information on prevalence), and thus only two variables enter the equation: the number of correctly diagnosed cases and the number of incorrectly diagnosed cases (here, 8 and 95, respectively). Furthermore, the computations are performed on natural numbers rather than on fractions. As can been in Fig. 1, all one has do to is simply divide 8 by the sum of 8 and 95 .

Second, it has been argued that the human mind is better tuned to processing information in the natural frequency format. Natural frequencies are the product of natural sampling (i.e., sequential encoding of events), and the central assumption is that for most of our evolutionary history, we have assimilated frequency information by encountering events case after case (e.g., Cosmides and Tooby, 1996; Gigerenzer and Hoffrage, 1995). The mathematical branch of probability was invented only in the mid-17th century (Daston, 1988) and only later became a representation method for risk and uncertainty. This can suggest that our minds are better adjusted to dealing with natural frequencies than with probabilities.

The results obtained by Gigerenzer and Hoffrage (1995) point to the usefulness of transforming statistical information into natural frequencies. Can we use this insight to improve nurses' ability to solve Bayesian inference tasks (such as in the mammography task)? Can health care professionals be trained to represent statistical information which is frequently expressed as probabilities or percentages (e.g., Gigerenzer et al., 1998) - in terms of natural frequencies?

The evidence suggests that training students in transforming statistical information into natural 
frequencies can be helpful. Several recent training studies have tested whether the natural frequency approach can lead to better results than the traditional probabilistic approach. Sedlmeier and Gigerenzer (2001) and Sedlmeier (1997) implemented this idea with a computer-based tutorial. In their study, university students were either trained to translate probabilities into natural frequencies or taught to insert probabilities into Bayes' rule and were subsequently asked to solve Bayesian frequency tasks (similar in nature to the mammography problem discussed above). Sedlmeier and Gigerenzer showed that subjects who were trained to translate probabilities into natural frequencies, compared with subjects who were trained to use Bayes' rule, had a better immediate learning effect (higher success rate), and a greater temporal stability of the improvement. In another study, Kurzenhäuser and Hoffrage (2002) examined whether teaching medical students to translate probability information into natural frequencies, in the classroom rather than in individual computer sessions, would yield similar results. They provided either a one-hour tutorial composed of traditional rule learning (i.e., Bayes' rule) or one that was designed to teach students about solving problems with natural frequencies. In a post-test two months after the training, $16 \%$ of the subjects who received the traditional Bayesian rule learning inferred the correct positive predictive value, whereas $47 \%$, or almost three times more, of the subjects who were trained in the natural frequency format were able to find the right answer. If medical students can improve, we see no reason why nurses cannot achieve similar results.

Much confusion in risk communication, in which risks are often communicated in probabilities or percentages, is due to the lack of understanding of what reference class the information expressed in percentages refers to (cf. Gigerenzer and Edwards, 2003). For instance, for a patient to understand a probability of $20 \%$ it needs to be clear from what class of events this percentage is taken from. The empirical findings presented here suggest that this confusion can be overcome: nurses, as important conveyors of health-related statistical information, could benefit from adopting a new method of dealing with statistical calculations by learning to translate probabilities into natural frequencies. By virtue of this competence nurses can enhance their ability to understand and effectively communicate statistical information and foster insight among their patients. As the studies reviewed here indicate, this competence can be educated. Therefore, instruction in techniques such as using natural frequencies that foster efficient communication should be part of the curriculum and continuing education for nurses.

Thus far we have discussed one possible solution while largely ignoring how the natural frequency format method could best be implemented in nurses' curriculum programs. One straightforward solution would be to employ Sedlmeier and Gigerenzer (2001) and Sedlmeier (1997) two-hour computerized tutorial - a tutorial that has been found, thus far, to yield the best learning results and effects (both in measures of accuracy and in stability). This tutorial includes all the necessary and relevant learning material required to translate probabilities into frequencies and thereafter to solve the Bayesian inference tasks.

Given that not all institutions have the necessary computer facilities, Kurzenhäuser and Hoffrage (2002) more traditional classroom setting tutorial could be used. Their one-hour tutorial contains four educational segments - including overhead slides and handouts - that can be easily implemented (with slight adaptation to meet the specific needs of nursing students) in nurses' statistical curriculums. Finally, Hoffrage et al. (2000) provide a simple and easily communicated four-step procedure to translate incidence, sensitivity, and specificity information stated in probabilities or percentages, as in the mammography example given above, into natural frequencies:

1. Select a population (e.g., 1000 women aged 40) and use the base rate to determine how many people in the population have the disease.

2. Take that result and use the test's sensitivity to determine how many individuals have the disease and how many received a positive test.

3. Take the remaining number of healthy people and use the test's false-positive rate to determine how many people do not have the disease but still test positive.

4. Compare the number obtained in step 2 with the sum of those obtained in steps 2 and 3 to determine how many people with a positive test actually have the disease. (p. 2261)

\section{Conclusion}

Enhancing patients' abilities to understand and utilize information is one of the goals of modern medical practice. Olsen and Gøtzsche (2001) urged women, as well as health professionals, to "consider [their] findings carefully when they decide whether or not to attend or support screening programs" (p. 1341). By learning to convert singleevent probabilities into natural frequencies and 
conveying probabilistic information in the natural frequency format, nurses can improve risk communication to their patients and, it is hoped, diminish anxiety and confusion levels surrounding various medical procedures. Educational programs for nurses need to include training in translating risk information which often come as probabilities, into natural frequencies. By thus making the reference class explicit, this method can increase the competency to evaluate risks more adequately and to weigh different risks against each other. Though at present we lack the empirical data needed to support the notion that nurses can benefit from our suggested program, we believe that the evidence discussed throughout this paper provides sufficient reason to draw attention to the benefits of using natural frequencies in nurses' practice as information providers.

Using natural frequencies, needless to say, cannot eradicate all the problems associated with statistical calculation and risk communication. As we have seen, however, the natural frequency format can facilitate making the correct inferences from statistical information, and nurses could capitalize on this effect both in their traditional and emerging roles. Given the importance of effective communication, changing the representation format can have a positive effect on patients' abilities to make informed decisions that are better aligned with their values and goals. It can improve, simultaneously, nurses' ability to tackle and solve statistical problems, resulting in more meaningful information for patients, who, in turn, are enabled to participate competently in shared decision making.

\section{Acknowledgements}

Financial support for this paper was provided by a Minerva fellowship to the first author, and by a Max Planck Society fellowship to the second author. We would like to thank Ulrich Hoffrage, Konstantinos V. Katsikopoulos, Stephanie Kurzenhäuser, Anita Todd and two anonymous reviewers for help with an earlier version. The authors contributed equally to the research and are listed alphabetically. Correspondence concerning this article should be addressed to either author via e-mail: yhanoch@mpib-berlin. mpg.de or pachur@mpib-berlin.mpg.de.

\section{References}

American Association of Colleges of Nursing n.d. Retrieved November 22, 2003. Available from <http://www.aacn. nche.edu/\%2Feducation\%2Fcareer.htm>.
Bush, P.J., Osterweis, M., 1978. Pathways to medicine use. Journal of Health and Social Behavior 19, 179-189.

Casscells, W., Schoenberger, A., Grayboys, T., 1978. Interpretation by physicians of clinical laboratory results. New England Journal of Medicine 299, 999-1000.

Chan, E., Sulmasy, D.P., 1998. What should men know about prostate-specific antigen screening before giving informed consent? American Journal of Medicine 105, 266-274.

Cosmides, L., Tooby, J., 1996. Are humans good intuitive statisticians after all? Rethinking some conclusions from the literature on judgment under uncertainty. Cognition 58, $1-73$.

Croyle, R.T., Lerman, C., 1999. Risk communication in genetic testing for cancer susceptibility. Journal of the National Cancer Institute Monographs 25, 59-66.

Daston, L.J., 1988. Classical Probabilities in the Enlightenment. Princeton University Press, Princeton.

DoH, 2003. Our Inheritance, Our Future - Realising the Potential of Genetics in the NHS. HMSO, London.

Eddy, D.M., 1982. Probabilistic reasoning in clinical medicine: problems and opportunities. In: Kahneman, D., Slovic, P., Tversky, A. (Eds.), Judgment Under Uncertainty: Heuristic and Biases. Cambridge University Press, Cambridge, pp. 249-267.

Edwards, A., Matthews, E., Pill, R., Bloor, M., 1998. Communication about risk: diversity among primary care professionals. Family Practice 15 (4), 296-300.

Ellis, P.M., Tattersall, M.H.N., 1999. How should doctors communicate the diagnosis of cancer to patients? Annals of Medicine 31 (5), 336-341.

Emery, J., Hayflick, S., 2001. The challenge of integrating genetic medicine into primary care. British Medical Journal 322, 1027-1030.

Erev, I., Cohen, B.L., 1990. Verbal versus numerical probabilities: efficiency, biases and the preference paradox. Organizational Behavior and Human Decision Making 9, 153-172.

Evans, J.P., Skrzynia, C., Burke, W., 2001. The complexities of predictive genetic testing. British Medical Journal 322, 1052-1056.

Gigerenzer, G., Edwards, A., 2003. Simple tools for understanding risks: from innumeracy to insight. British Medical Journal 327, 741-744.

Gigerenzer, G., Hoffrage, U., 1995. How to improve Bayesian reasoning without instruction: frequency format. Psychological Review 102 (4), 684-704.

Gigerenzer, G., Hoffrage, U., Ebert, A., 1998. Aids counselling for low-risk clients. Aids Care 10, 197-211.

Hallowell, N., 2000. A qualitative study of the information needs of high-risk women undergoing prophylactic oophorectomy. Psycho-Oncology 9, 486-495.

Hallowell, N., Statham, H., Murton, F., Green, J., Richards, M., 1997. "Talking about chance": the presentation of risk information during genetic counseling for breast and ovarian cancer. Journal of Genetic Counseling 6, 269-286.

Hoffrage, U., Gigerenzer, G., Krauss, S., Martignon, L., 2002. Representation facilitates reasoning: what natural frequencies are and what they are not. Cognition 84, 343-352.

Hoffrage, U., Kurzenhäuser, S., Gigerenzer, G., in press. Understanding benefits and risks: Why the representation of statistical information matters. In: Bibace, R., Laird, J., Noller, K. D., Valsiner, J. (Eds.), Heuristics and values: Relating the Universals and the Particulars in Thinking About Health. Greenwood Publishing Group, Westport, CT.

Hoffrage, U., Lindsey, S., Hertwig, R., Gigerenzer, G., 2000. Communicating statistical information. Science 290, 2261-2262. 
Hofman, K.J., Tambor, E.S., Chase, G.A., Geller, G., Faden, R.R., Holtzman, N.A., 1993. Physician's knowledge of genetics and genetic tests. Academic Medicine 68, 625-632.

Howie, J.G.R., Heaney, D.J., Maxwell, M., Walker, J.J., Freeman, G.K., Rai, H., 1999. Quality at general practice consultations: cross sectional survey. British Medical Journal 319, 738-743.

Kirk, M., McDonald, K., Longley, Iredale R., 2003. Fit for practice in the genetics era: Defining what nurses, midwives and health visitors should know and be able to do in relation to genetics. Genomics Policy Unit, University of Glamorgan.

Kurzenhäuser, S., Hoffrage, U., 2002. Teaching Bayesian reasoning: an evaluation of a classroom tutorial for medical students. Medical Teacher 24 (5), 516-521.

Lashley, F.R., 1999. Genetic testing, screening, and counseling issues in cardiovascular disease. The Journal of Cardiovascular Nursing 13 (14), 110-126.

Lea, D.H., 2000. A clinician's primer in human genetics. Nursing clinics of North America 35 (3), 583-614.

Lea, D.H., Tinley, S.T., 1998. Genetics in the OR: implications for perioperative nursing practice. AORN Journal 67 (6), $1175-1180$.

Lessick, M., Anderson, L., 2000. Genetic discoveries: challenges for nurses who care for children and their families. Journal of the Society of Pediatric Nurses 5 (1), 47-51.

Ley, P., 1982. Satisfaction, compliance, and communication. British Journal of Clinical Psychology 21, 241-254.

Lidz, C.W., Meisel, A., Zerubavel, E., 1984. Informed Consent: A Study of Decision Making in Psychiatry. The Guilford Press, New York.

Lipkus, I.M., Samsa, G., Rimer, B.K., 2001. General performance on a numeracy scale among highly educated samples. Medical Decision Making 21, 37-44.

Maguire, P., 1999. Improving communication with cancer patients. European Journal of Cancer 35 (14), 2058-2065.

McManus, R.J., Mant, J., Meulendijks, C.F.M., Salter, R.A., Pattison, H.M., Roalfe, A.K., Hobbs, F.D.R., 2002. Comparison of estimates and calculations of risk of coronary heart disease by doctors and nurses using different calculation tools in general practice: cross sectional study. British Medical Journal 324, 459-464.
Newell, G.R., Vogel, V.G., 1988. Personal risk factors: what do they mean? Cancer 62 (8), 1695-1701.

Olsen, O., Gøtzsche, P.C., 2001. Cochrane review on screening for breast cancer with mammography. Lancet 358, 340-342.

Reyna, V.F., 2001. Genetic testing and medical decision making. Archive of Internal Medicine 161, 2406-2408.

Schwartz, L.M., Woloshin, S., Black, W.C., Welch, G., 1997. The role of numeracy in understanding the benefit of screening mammography. Annals of Internal Medicine 127, 966972.

Sedlmeier, P., 1997. BasicBayes: a tutor system for simple Bayesian inference. Behavioral Research Methods, Instruments, and Computers 29, 328-336.

Sedlmeier, P., Gigerenzer, G., 2001. Teaching Bayesian reasoning in less than two hours. Journal of Experimental Psychology: General 3, 380-400.

Stoltzfus, P.K., Rust, D., Reid, T., 2001. Molecular cytogenetics and gene analysis: implications for oncology nurses. Clinical Journal of Oncology Nursing 5 (5), 201-206.

Taylor, S., 2002. Health Psychology, fifth ed. McGraw-Hill, New York.

Taylor, S., Muncer, S., 2000. Redressing the power and effect of significance. A new approach to an old problem: teaching statistics to nursing students. Nurse Education Today 20, 358-364.

Tzeng, H.M., Ketefian, S., 2003. Demand for nursing competencies: an exploratory study in Taiwan's hospital system. Journal of Clinical Nursing 14 (4), 509-518.

Valanis, B., 2000. Professional nursing practice in an HMO: the future is now. Journal of Nurse Education 39 (1), 13-20.

Wear, S., 1993. Informed Consent: Patient Autonomy and Physician Beneficence within Clinical Medicine. Kluwer Academic Publishers, Dordrecht.

Weinstein, N.J., 1999. What does it mean to understand a risk? Evaluating risk comprehension. Journal of the National Cancer Institute Monographs 25, 15-20.

Williams, J.K., 1998. Genetic testing: implications for professional nursing. Journal of Professional Nursing 14 (3), 184-188.

Wivell, G., Denton, E.R.E., Eve, C.B., Inglis, J.C., Harvey, I., 2003. Can radiographers read screening mammograms. Clinical Radiology 58, 63-67.

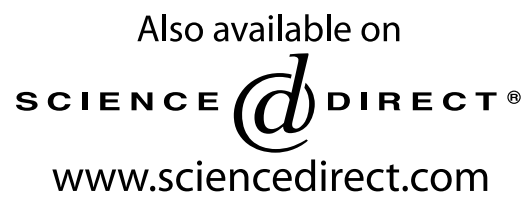

